\title{
Visual imagery in backward masking
}

\author{
ADAM REEVES \\ Institut für Arbeitsphysiologie, D-4600 Dortmund 1, West Germany
}

\begin{abstract}
Observers viewed briefly presented target dot patterns, either at low contrast without a mask (no mask, or NM) or at high contrast and followed by a long-lasting patterned mask (backward masking, or BM). Experiment 1 demonstrated independent processing of NM target dots but limited capacity processing of BM target dots. Experiments 2 and 3 showed that visual images may radically change sensitivity $\left(d^{\prime}\right)$ in $B M$ but not in NM. Results suggest that $d^{\prime}$ is reduced if the image suppresses dots relevant for the detection task, but that $d^{\prime}$ is raised if the image suppresses dots that compete for processing with those the observer must detect.
\end{abstract}

Visual stimuli may have their appearances modified by concurrent visual imagery (Segal, 1971) or, under appropriate conditions, be reported as entirely imaginary (Perky, 1910). Segal and Fusella (1970) found that sensitivity $\left(d^{\prime}\right)$ to a visual target presented on a Ganzfeldlike background was reduced by .69 by concurrent visual imagery but by only .37 by concurrent auditory imagery. (Auditory imagery reduced sensitivity to a tone more than did visual imagery, in a similar fashion.) Thus the effect of an image on identification and detection of targets is at least partly modality specific. Visual imagery might lower visual sensitivity merely by inducing changes in eye position or in pupil size, but imagery lowered $d^{\prime}$ when photography of the eye showed that no such changes were occurring (Reeves \& Segal, 1973). These facts make it likely that visual imagery influences the visual system either retinally or postretinally; but it is not known at what stage of processing such influences might take effect. The present research tackles this question.

Current theories postulate two initial stages in the processing of multielement displays, a registration stage and a scanning stage. The registration stage is a fast parallel operation in which all positions in the visual field are processed at once and independently of one another. Registered display elements are held in a visual information store (Sperling, 1963) which may outlast the display if the latter is brief. The second stage, a slower, limited-capacity operation, scans the elements in the store, either in a strict serial fashion at about $10 \mathrm{msec} /$ item (Sperling, 1963) or in an overlapping manner at the same average rate. Masking by noise can disrupt the first (registration) stage, while masking by an aftercoming pattern can disrupt the second stage (Turvey, 1973). Subsequent stages may be responsible

This research was carried out at the City University of New York and was supported in part by a CUNY research grant to Dr. Philip Liss. The author wishes to thank Dr. Liss, Dr. Sydney Segal, and Dr. Gertrude Schmeidler for valuable discussion. The author's present address is: Institut für Arbeitsphysiologie, Postfach 15 08, D-4600 Dortmund 1, West Germany. for a nonmaskable short-term visual memory of the target (Philips, 1974) on which higher figural processes may operate (Turvey, 1973, p. 43). The aim of the present research was to isolate the first two stages and discover whether or not visual imagery might influence them.

Stage isolation may be achieved by the correct choice of masking condition and demonstrated by the effect of target numerosity-the number of distinct elements in the target display-on performance. Parallel independent processing of target elements in the first stage means that if performance is governed by the first stage alone, the probability of any target item being detected must be independent of the number of target elements. Such independence has been found when letters were presented briefly without a mask (the "no-mask" condition) or concurrently with a mask (Liss, 1968), or without a mask in a cluttered visual field if the elements are more than $.5 \mathrm{deg}$ apart (Gardner, 1973). Limited capacity processing in the second stage means that if performance is governed by the second stage, the probability of any target element's being detected must go down if the number of target elements is increased beyond a limit determined jointly by the processing rate and the time available for processing. Such results have been obtained when target energy is enough for successful registration (in Stage 1), but the time available for processing is limited by an aftercoming patterned mask (the backward-mask condition) (Liss, 1968; Sperling, 1963; Turvey, 1973; reviews by Kahneman, 1968; Scheerer, 1973).

Both independence and limited-capacity effects of target numerosity on performance may be demonstrated with targets constructed from dots (displayed at least $.5 \mathrm{deg}$ apart to avoid lateral interactions). Uttal (1975, pp. 35-39) has catalogued the advantages of dot targets for isolating processing stages. When dot targets are shown briefly without a mask (the no-mask, or NM, condition), the probability of detecting any particular dot is independent of the number of dots presented. (Uttal, 1975, p. 6 gives references.) On the other hand, when the target is shown with high contrast, but is 
followed by a patterned mask (the backward-masking, or BM, condition), limited capacity effects can be shown. The first experiment demonstrates these effects, and subsequent experiments introduce visual imagery in the same conditions.

\section{GENERAL METHOD}

\section{Observers}

Observers were City University of New York undergraduates with no prior experience with visual experiments. They had normal or corrected-to-normal vision. They were paid independently of their results. Different observers were used in each experiment: four, five, and four in Experiments 1, 2, and 3, respectively. Two potential observers were discarded because they reported being unable to evoke a simple standard image (a cross) while looking with eyes open at a blank gray wall.

\section{Apparatus and Stimuli}

Stimuli were presented binocularly by a Harvard-type Gerbrands three-field tachistoscope. The fields provided the target, the mask, and a fixation point. Luminances of white cards in all three fields were matched by eye and were $3.5 \mathrm{~mL}$ as measured by a MacBeth illuminometer. The stimuli were white index cards which subtended $7.4 \times 11.1 \mathrm{deg}$ at the eye. Targets were 5 -mm-diam ( $.36 \mathrm{deg}$ ) Chart-Pak matt black dots mounted on white index cards at the corners of an imaginary square which subtended $3.4 \mathrm{deg}$ on each side. A $1-\mathrm{mm}$ fixation point was positioned at the center of the imaginary square. The mask was four black $6-\mathrm{mm}$ Chart-Pak dots $(.44 \mathrm{deg})$ placed one in each corner of the same square. The slightly larger dots of the mask always covered the target dots despite the free play allowed by the card holder of the tachistoscope. In Experiment 3, target and mask dots were mounted around the same standard imaginary square, but in a more complex pattern, as described in the procedure section of that experiment.

\section{Procedure}

The observer viewed the fixation field and, when properly fixated, initiated a trial by means of a buttonpress. After a $1-\sec$ wait, timing circuitry presented the stimulus sequence by flashing the light sources in each field, as shown in Figure 1 (inset). In the no-mask (NM) condition, the target was presented at an adjustable luminance for a nominal $4 \mathrm{msec}$. The target was then replaced once more by the fixation field. In the backward-mask (BM) condition, the target was presented for a nominal $20 \mathrm{msec}$ at the standard $(3.5 \mathrm{~mL})$ intensity. It was followed by the fixation field, also at the standard intensity, for a variable interval (the ISI). The mask was then presented for a nominal $200 \mathrm{msec}$ and followed once more by the fixation field. The time from target onset to mask onset, the stimulus onset asynchrony (SOA), was controlled by varying the ISI.

The task of the observer was to report the target "identity" on every trial, as detailed separately for each experiment. Decision latency was not recorded. Observers were told to be as accurate as possible, to take as long as they needed, and to guess roughly at random among the report possibilities if they were totally unsure. Observers were not given feedback of any sort, except that during the initial practice trials they were shown the target to help orient them to the task.

\section{Imagery Instructions}

Observers were told to have no image at all during no-imagery trials and to have a specified image during imagery trials. They were allowed to rerun a trial if imagery had occurred during no imagery or if their image had faded during imagery. No imagery meant both that observers were asked to avoid voluntary imagery and that, if they noticed afterimages from stimulus dots or from the illuminated field, they were to wait until such images had cleared. Imagery was trained by showing observers the stimulus they were to imagine. They were asked to correctly locate the image in space and to give it the same number and shape of dots as the stimulus shown. Such images were described as spatially faithful and projected onto the plane of the tachistoscope field but were less intense than the stimulus shown. The stimuli to be imagined are described in the procedure section of each experiment, but they always included the mask itself, whose shape was visible on every trial in masking: other shapes were shown at the start of every relevant block of trials to help remind the observer of what was to be imagined.

\section{Design}

The time from target onset to mask (the SOA) was set individually for each observer during initial practice. The SOA was progressively reduced until the observer was performing at a fixed level of accuracy, as detailed in the procedure section for each experiment. Target luminance was adjusted likewise in the NM condition. SOAs and target luminances were then fixed for the remainder of the session. When observers ran for a second day of testing, the SOAs and target luminances were determined again in the same manner.

Conditions were run in blocks of fixed length ( 16 or 20 trials). In each block, the conditions of imagery, mask, and target type were held constant. Conditions were changed every block, according to different orders as specified below in each experiment. Observers rested for about $1 \mathrm{~min}$ between blocks. They received a minimum of 48 practice trials in each masking condition on each day.

\section{EXPERIMENT 1}

The purpose of this experiment was to demonstrate independence and limited capacity effects in NM and $\mathrm{BM}$, respectively, under the no-imagery instruction.

\section{Procedure}

The targets had from zero to four dots. They were arranged, by numerosity, into five sets. The dots were located at the corners of the standard imaginary square (see General Method). The single dot in the one-dot set and the missing dot in the three-dot set were located in a different corner on each of the four cards of each set. Of the six possible different arrangements of two dots in four corners, four were chosen to make up the two-dot set. The SOAs chosen in initial practice were between 45 and $65 \mathrm{msec}$ and averaged $50 \mathrm{msec}$ over the four observers used.

The observer's task was to report on each trial how many dots he had seen, as far as possible without guessing. The observers knew that the targets were equally likely to have zero to four dots. They were given the no-image instructions detailed in the General Methods section. On each trial, a target was chosen without replacement and at random from the group of 20 targets. Mask conditions (BM and NM) were varied every block of 20 trials in an ABBA fashion, with two observers receiving NM first. Each observer received 80 trials per condition of mask and target numerosity. No trials were terun.

\section{Results}

The mean number of dots reported is plotted in Figure 1 against the number of dots presented on each target. Crosses indicate the NM condition and dots, the BM condition. Bars show the range of the data for the four observers tested. The straight line at $\mathbf{4 5} \mathrm{deg}$ shows perfect performance. The $\mathrm{BM}$ results rise along this 


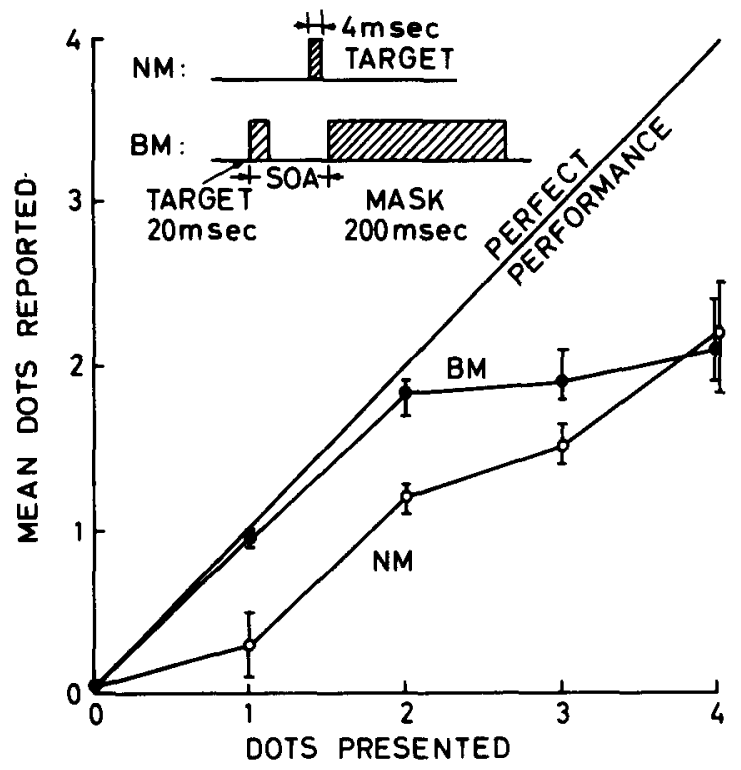

Figure 1. Mean number of dots reported as a function of the number presented, in no masking (circles) and backward masking (dots). Bars show the range for the four observers. Up to four dots were presented at the four corners of an imaginary square (3.6 deg on its side) in target cards. The mask was all four dots. The inset shows the temporal sequence in no masking (NM) and backward masking (BM). Targets were $4 \mathrm{msec}$ in NM and $20 \mathrm{msec}$ in BM. The BM mask was $200 \mathrm{msec}$ and followed the target. Time from target onset to mask onset is the stimulus onset asynchrony (SOA). Target luminance in NM and SOA in BM were varied to achieve criterion performance levels.

line and then level off at about 2.0 dots reported. The NM results rise along a line of about slope .5. The two curves meet at four dots presented because SOAs and target luminances (in BM and NM, respectively) were so chosen during practice. The difference between the 1.85 dots reported in $\mathrm{BM}$ and the 1.20 reported in NM, when two dots were presented, is significant $[\mathrm{t}(3)=$ $2.70]$ at $5 \%$ in a one-tailed $t$ test. ${ }^{1}$

\section{Discussion}

The parallel independent model predicts that the mean number of dots reported, NR, is proportional to the number presented, NP. This prediction was tested by finding a factor, $M$, in the equation $N R=M \cdot N P$ which minimized the squared error of prediction of NR. The proportion of variance of NR accounted for by this equation is $\mathrm{r}^{2}$, the square of the Pearson productmoment correlation between observed and predicted NRs. In the NM condition, $\mathrm{r}^{2}$ was greater than .93 for each of the four observers (it averaged .935). The factor M lay between .45 and .59 and averaged .53 . In the BM condition, however, $\mathrm{r}^{2}$ lay between .65 and .91 for the different observers and averaged .80 . Thus, the parallel independent model provides a good description of the NM data, but not of the BM data. In $\mathrm{NM}$, remaining deviations from the model were essentially unsystematic; for example, mean $r^{2}$ for a quadratic regression model $\left(\mathrm{NR}=\mathrm{M}_{2} \cdot \mathrm{NP}^{2}+\mathrm{M}_{1} \cdot \mathrm{NP}+\mathrm{M}_{3}\right)$ rose insignificantly to 941 .

The limited-capacity model may be expressed in several forms. The simplest assumes that the observer can process up to A dots without error, before the mask arrives, and then can process no more. The value of $A$ is determined by the SOA, which, in this study, was fixed. Hence, NR = Min(A,NP), with A constant. This simple model generated predictions with $\mathbf{r}^{2}$ better than .987 for each observer for the BM data. The model failed for NM data, with $\mathrm{r}^{2}$ between .59 and .65 for each observer. More sophisticated models might either assume that $\mathbf{A}$ was a random variable or that overlapping processing was occurring. In either case, the plot of NR against NP would show a smoother inflection point. These ideas suggest a quadratic fit. The $r^{2}$ for the quadratic regression model was greater than .96 for each observer and averaged .984 , with three free parameters. Clearly, the data do not exclude such a model, although the model with an abrupt cutoff at the $A$ dots might be preferred for its simplicity. Values of A lay between 1.8 and 2.25 dots and averaged 1.98 dots.

\section{EXPERIMENT 2}

Imagery and no imagery were compared in NM and BM for targets of varying numerosity in this experiment.

\section{Procedure}

The targets were changed to take advantage of a yes-no procedure. Two target sets were used: the low-numerosity set, which contained either zero or one dot, and the high-numerosity set, which contained either three or four dots. The dot in the one-dot card and the missing dot in the three-dot card always occurred in the lower left corner of the standard imaginary square. These cards are illustrated in the upper panel of Figure 2. The SOAs chosen after initial practice ranged between 40 and $70 \mathrm{msec}$ and averaged $52 \mathrm{msec}$.

The observer's task was to report on each trial whether the lower left dot had been present or absent in the target. He knew that either possibility was equally likely. He was told to ignore as far as possible any additional target dots that he saw appearing in other corners of the imaginary square. Correct (central) fixation was emphasized. Observers were given both imagery and no-imagery instructions, as detailed in the General Method section. The image they were required to hold in mind in the imagery condition was the four-dot mask.

Observers received 64 trials in each condition they ran. Conditions were changed after every 16-trial block. The imagery instruction was alternated every block. Of the five observers, two were given NM on Day 1 and BM on Day 2, two were given the reverse order, and one received the BM condition alone, as he did not return on Day 2.

\section{Results and Discussion}

A report of the presence of the lower left dot was scored as a hit if a dot had been present and as a false alarm if not. The corresponding frequencies, $f_{h i t}$ and $f_{f a}$, were converted to sensitivity $\left(d^{\prime}\right)$ by the formula $\mathrm{d}^{\prime}=\mathrm{z}\left(\mathrm{f}_{\mathrm{hit}} / 64\right)-\mathrm{z}\left(\mathrm{f}_{\mathrm{fa}} / 64\right)$, on the assumption that the unit variance normal distribution $z$ describes the dis- 


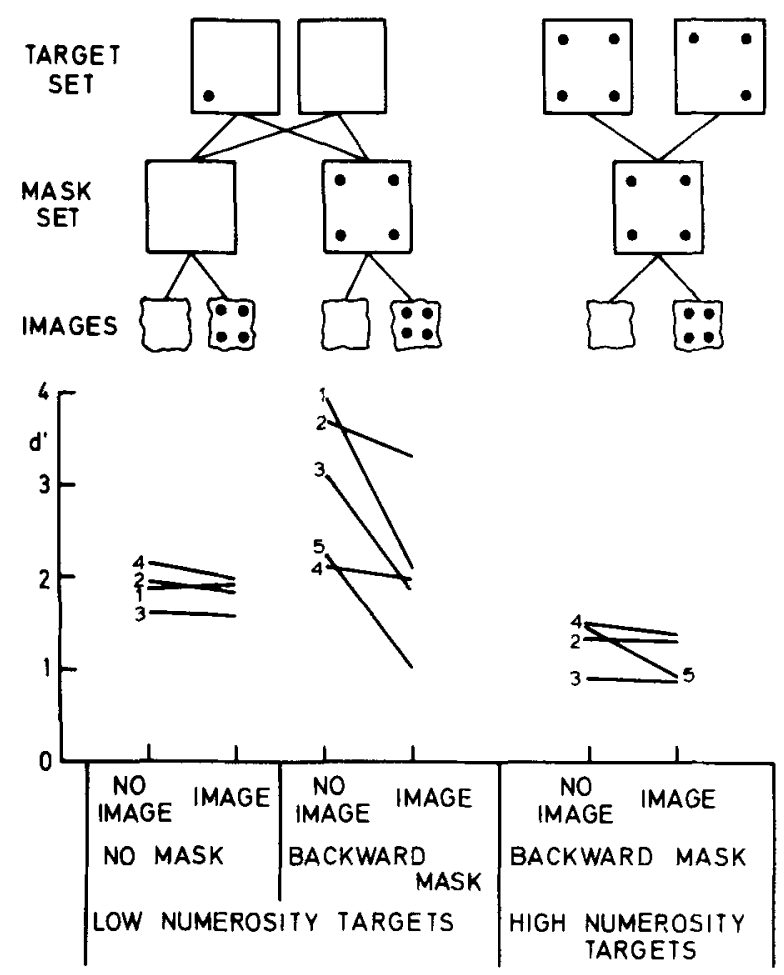

Figure 2. The upper panel shows the low-numerosity target set on the left and the high-numerosity set on the right. Low-numerosity targets were presented with no mask or with a backward mask of four dots; high-numerosity targets were presented only with the backward mask. The lower row of the upper panel shows the images requested of the observers; they correspond to the conditions shown in the lower panel. The lower panel shows d' for each of five observers in each of the six conditions identified on the abscissa and represented vertically above in the upper panel.

tributions of strengths on both signal (dot present) and noise (dot absent) trials.

Sensitivities in each condition of target numerosity, imagery, and mask are shown in the lower panel of Figure 2, underneath a pictorial representation of the same condition in the upper panel. The four conditions under masking were analyzed by a two-way fixed effects ANOVA (Hays, 1963). The main effect of imagery was significant $[F(1,16)=4.5, p<.05]$, as was that of numerosity $[\mathrm{F}(1,16)=22.5, \mathrm{p}<.01]$. Analyzing the data of each observer separately, the null hypothesis that all $\mathrm{d}^{\prime}$ values were equal was tested by computing the statistic $U_{o}^{\prime}$ (Marascuilo, 1970, p. 241), which has a chi-square distribution with $5 \mathrm{df}$. The null hypothesis was rejected at the $1 \%$ significance level for four of the five observers (the exception being Observer 4). Values of response bias, $\beta$ (the ratio of hit density to false alarm density), reading from left to right in Figure 2, were $1.66,1.84,1.90,1.65,1.15$, and 1.39 (averaged over observers). Mean $\beta$ was 1.63 in imagery and 1.57 in no imagery $[\mathrm{t}(13)=.21$; n.s.), confirming Segal and Fusella (1970), who found that visual imagery did not change $\beta$.

Increasing the number of target dots in the BM condition lowered sensitivity, the result to be expected from
Experiment 1. For example, in the no-image BM condition, $\mathrm{d}^{\prime}$ was 3.04 in low numerosity and 1.33 in high. The new result was that the four-dot image decreased $\mathrm{d}^{\prime}$ in the low-numerosity BM condition, for all five observers, but had virtually no effect in other conditions. The differences in $d^{\prime}$ between the imagery and the no-imagery conditions in low-numerosity $\mathrm{BM}$ were tested by planned comparisons in the two-way ANOVA and found to be significant $[\mathrm{t}(16)=1.82, \mathrm{p}<.05]$. Taking each observer separately, the differences in $d^{\prime}$ were tested by the $\mathrm{Z}$ test of Marascuilo (1970, p. 240) and found to be significant at the $1 \%$ level for Observers 1, 3, and 5, but insignificant for Observer 2 .

It might be argued that the values of $\mathrm{d}^{\prime}$, calculated under the equal variance assumption, could be severely biased if that assumption were false. Moreover, criterion shifts alone might lead to spurious changes in $\mathrm{d}^{\prime}$, as calculated under an incorrect assumption concerning the underlying variances. In fact, Segal and Fusella (1970) found the ratio of the signal standard error, $\sigma_{\mathrm{s}}$, to the noise, $\sigma_{\mathrm{n}}$, was not unity but, rather, varied between 2.3 and 2.6 in various conditions of visual discrimination similar to NM. The ratio did not vary when imagery was added to the task. (These ratios were estimated as the inverses of the slopes of the ROC curves shown in their Figures 1 and 2, ignoring data points with false alarm rates less than $1 \%$ based on 128 trial sessions.) The data of Experiment 2 were reanalyzed, with the assumption that the ratio $\sigma_{\mathrm{s}} / \sigma_{\mathrm{n}}$ was 2.3 in all conditions. Letting $\mathrm{d}_{\mathrm{s}}$ be the difference between the means of the assumed underlying signal and noise distributions, divided by the average of $\sigma_{\mathrm{s}}$ and $\sigma_{\mathrm{n}}$ (Treisman, 1977), elementary geometry based on his Figure 1 shows that

$$
d_{s}=\sqrt{h^{2}+f^{2}} \sqrt{2} \sin \left[\tan ^{-1}\left(\sigma_{s} / \sigma_{n}\right)-\tan ^{-1}(h / f)\right]
$$

where $h=z\left(P_{h i t}\right)$ and $f=z\left(P_{f_{a}}\right)$. Using this calculation, values of $d_{s}$ were essentially the same as the values of $d^{\prime}$ found before on the equal variance assumption. That is, $\mathrm{d}_{\mathrm{s}}$ did not differ from $\mathrm{d}^{\prime}$ by more than .3 in any condition for any observer; and, averaging over observers, $d_{s}$ was within $4 \%$ of $d^{\prime}$ in each of the six conditions of Experiment 2.

The lack of an effect of the image in the NM condition is evidence that imagery per se does not reduce performance. However, a small effect might have been expected here, since Segal (1971) has repeatedly found small effects of imagery in NM conditions (e.g., Segal $\&$ Fusella, 1970, found an effect of $.24 \mathrm{~d}^{\prime}$ units). It may be that the present research used too few observers to pick up such a small effect. On the other hand, Segal's images were complex (e.g., an elephant) and may have disrupted processing only at higher levels. In that case, one might conclude that imagery has no effect on stage 1 processing.

The image had little effect in high-numerosity BM conditions but had a large effect in low-numerosity conditions. This result may be explained if the imaged 
dots take up some processing capacity in the second stage. The reduction in sensitivity normally obtained by increasing the number of target dots in BM may, to some extent, be obtained with imaginary target dots. Presumably, imaged dots summate with target dots in the high-numerosity condition and so have little further effect on second-stage processing. But in low-numerosity conditions, imaged dots appear where target dots do not and so take some processing capacity away from the critical lower left corner. It must be assumed that additional imaged dots, like additional target dots, cannot be totally ignored, even though the observer knows exactly where they are located. (This does not imply, however, that no attentive selection is occurring-merely that the additional dots cannot be excluded entirely.)

\section{EXPERIMENT 3}

To test further the theory that images may take up second-stage processing capacity, target, image, and mask patterns were chosen to provide a larger range of possible outcomes. In particular, some combinations of patterns were expected to show decrements in performance with imagery, as obtained before, but other combinations were expected to show improvements in performance. Such improvements were expected if the image suppressed target elements that otherwise would have taken up second-stage processing capacity.

\section{Procedure}

The critical difference in this experiment was the use of more complex targets and masks; these are shown in the upper panel of Figure 3. The mask consisted of 12 dots mounted in four groups of 3 dots each, at the top, bottom, left, and right of the standard imaginary square. The 3 dots of each group formed an L shape. The dots were $.58 \mathrm{deg}$ from their nearest neighbors (center to center). The dots them selves had diameters of $.44 \mathrm{deg}$ (mask) and .36 deg (target), as before. The four L-shaped mask dot-triplets were oriented as shown in Figure 3. Target cards contained 8 dots arranged as four dot-pairs in positions exactly covered by mask triplets. A target dot-pair could lie under the vertical pair of the mask triplet or under the horizontal. The targets were in two sets. The horizontal set $(\mathrm{H})$ contained equal numbers of targets with only horizontal dot-pairs, and targets with three horizontal dot-pairs and one vertical. The vertical set (V) was similar: equal numbers of targets with all four vertical and with three vertical and one horizontal dot-pair. The critical dot-pair, which was oriented at 90 deg to the remainder of the dot-pairs, could occur equally often in any of the four positions (top, bottom, left, and right of the square). Examples are shown at the top of Figure 3. Note that the union of the targets is equal to the mask, as in previous experiments.

The observer's task was to report on every trial whether the target had been "same," that is, with all its dot-pairs in the same orientation, or "different," that is, with one dot-pair in the opposite orientation from the rest. Targets were shown only in the BM condition. After initial practice, SOAs were fixed at $120 \mathrm{msec}$ for one observer and at $170 \mathrm{msec}$ for three others; at these SOAs, they were approximately $80 \%$ correct in assigning "same" and "different" judgments under the no-imagery instruction. Imagery instructions were as detailed in the General Methods section above. The stimulus to be held in mind during imagery was; the four vertical dot-pairs (image V), or the four horizontal dot-pairs (image $\mathrm{H}$ ), or the 12-dot mask (image $M$ ), or nothing (no image). Although these images are more complex than those
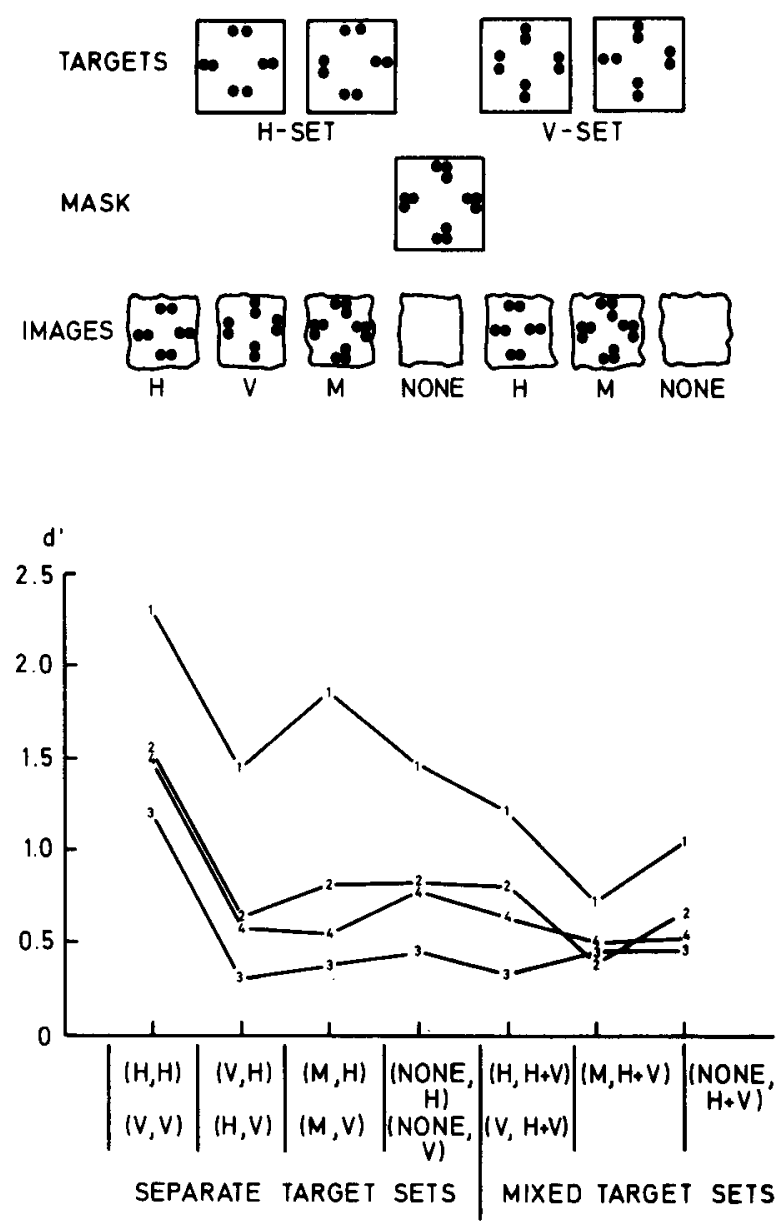

Figure 3. The upper panel, upper row shows two examples each of the $H$ and $V$ target sets; one example of each set has a dot-pair oriented at 90 deg to the other three dot-pairs. The observer discriminated "same"' targets (with all dot-pairs of the same orientation) from "different" dot-pairs (with one critical dot-pair reoriented). The critical dot-pair could occur in any of the four positions. The 12-dot mask, which is a union of all the targets, is shown below. Targets were always followed by the mask (the BM condition). The upper panel, bottom row shows images that were requested of the observer in the condition shown in the lower panel. The lower panel shows d' for four observers as a function of the condition indicated on the abscissa. Conditions are identified in (image, target) format, so that $(\mathbf{M}, \mathrm{H})$ means an image of the mask paired with the $H$ target set. When two conditions were averaged together, they are indicated one above the other below the abscissa. (The image of the upper condition is shown in the bottom row of the upper panel.)

used before, the observers did not seem to find them harder to maintain. The proportion of trials rejected because of imagery failures was similar $(2.5 \%$ in Experiment 2 and $3.1 \%$ in Experiment 3).

Targets were presented in three different conditions: the horizontal set alone (H), the vertical set alone (V), and both sets mixed together in a random order $(H+V)$. In the $H$ and $\mathrm{V}$ conditions, the observer knew the orientation of the critical dot-pair on which his discrimination was based (this was explained to all observers). In the $\mathrm{H}+\mathrm{V}$ condition, he did not: correct performance meant that all four dot-pairs had to be fully processed without error.

The 4 image conditions were crossed with the 3 conditions of target presentation to yield 12 conditions. The conditions were changed every block of 16 trials according to a different pseudo- 
random order for each observer. Observers received 64 trials per condition.

\section{Results}

Responses of "same" were scored as hits if the target had contained dot-pairs that were all oriented the same way, and as false alarms otherwise. Hit and false alarm rates were converted to sensitivities ( $\left.d^{\prime}\right)$, as in Experiment 2. The data were then reduced by averaging together pairs of like conditions, on the assumption that vertical and horizontal dot-pairs (and images) did not intrinsically differ from each other. Denoting a condition by (image, target) [e.g., $(\mathrm{H}, \mathrm{V})$ denotes trials with $\mathrm{H}$ imagery and a $\mathrm{V}$ target], $(\mathrm{H}, \mathrm{H})$ was averaged with $(\mathrm{V}, \mathrm{V}) ;(\mathrm{H}, \mathrm{V})$ with $(\mathrm{V}, \mathrm{H}) ;(\mathrm{M}, \mathrm{H})$ with $(\mathrm{M}, \mathrm{V})$; (none,H) with (none, $\mathrm{V}$ ); and $(\mathrm{H}, \mathrm{H}+\mathrm{V})$ with $(\mathrm{V}, \mathrm{H}+\mathrm{V})$. Mean d's did not differ by more than .27 units between the members of each pair, so the averaging assumption is probably valid.

Mean d's are shown in the lower panel of Figure 3 for each of the seven conditions left after averaging. Different symbols show the four observers. The conditions are shown on the abscissa in the (image, target) notation, with the two averaged conditions shown one on top of the other. (The image associated with the upper condition is displayed vertically above in the upper panel of the figure.)

Averaged over the four observers, values of $\beta$ were $1.31,1.09,1.42,1.18,1.12,1.05$, and 1.16 in the seven conditions plotted from left to right in Figure 3. Averaging these $\beta$ s over target sets, mean $\beta$ was 1.17 for image $\mathrm{H}$ or $\mathrm{V} ; 1.23$ for image $\mathrm{M}$; and 1.17 for no image: a $t$ test of the difference between image $M$ and no image showed $\mathrm{t}(7)=.54$ (n.s.). The lack of response bias ( $\beta$ close to 1.0) reflects the forced-choice nature of the "same" vs. "different" task. When $\beta=1.0$, estimates of $\mathrm{d}$ ' are independent of the slope of the ROC curve and hence of the assumed $\sigma_{\mathrm{s}} / \sigma_{\mathrm{n}}$ ratio. When that was set to 2.4 and $d_{s}$ was found according to the equation given in the discussion of Experiment 2, values of $d_{s}$ were always within .18 units of $\mathrm{d}^{\prime}$ for every observer and condition of Experiment 3.

A two-way fixed effects ANOVA ${ }^{2}$ was applied to the $d^{\prime}$ scores. Target set (separate or mixed) and imagery instruction (none, mask, "same," and "different") were both significant as main effects $[F(1,24)=5.97$, and $\mathrm{F}(3,24)=3.23$, respectively] at the $5 \%$ level. Analyzing the results for each observer separately, the hypothesis that all d's were equal was rejected at the $5 \%$ level by the chi-square test of Marascuilo (1970, p. 241) for three observers, and at the $10 \%$ level for the fourth.

The baseline conditions are those with no image: (none, $\mathrm{H}$ ), (none, $\mathrm{V}$ ), and (none, $\mathrm{H}+\mathrm{V})$. (None, $\mathrm{H}$ ) and (none, $\mathrm{V}$ ) had mean $\mathrm{d}$ 's of .88 , on average, and (none, $\mathrm{H}+\mathrm{V}$ ) had a mean $\mathrm{d}^{\prime}$ of .66 . The difference presumably stems from the fact that, in (none, $\mathrm{H}$ ) and (none, $\mathrm{V}$ ), the observer knew the orientation of the critical dot- pair, but in (none, $\mathrm{H}+\mathrm{V}$ ) he did not. These baseline conditions may be compared with those in which the observer imaged a mask (M) to reveal the effects of imagery per se on sensitivity. Mean $\mathrm{d}^{\prime}$ s were .89 in the $(\mathrm{M}, \mathrm{H})$ and $(\mathrm{M}, \mathrm{V})$ conditions and .50 in $(\mathrm{M}, \mathrm{H}+\mathrm{V})$. On average, these values are only $.08 \mathrm{~d}$ ' units lower than in the baseline conditions. Thus, imagery per se had very little effect on sensitivity. (The high numerosity BM condition of Experiment 2 is comparable.)

When the image was related specifically to the target pattern, however, changes in sensitivity occurred. For all four observers, sensitivity was highest in the $(\mathrm{H}, \mathrm{H})$ and $(\mathrm{V}, \mathrm{V})$ conditions: mean $\mathrm{d}^{\prime}$ was 1.66 , in comparison with the baseline (none, $\mathrm{H}$ ) and (none, $\mathrm{V}$ ) conditions, which had a mean $\mathrm{d}^{\prime}$ of .88 . This difference was significant when tested by a planned comparison in the twoway ANOVA $^{2}[\mathrm{t}(24)=1.83, \mathrm{p}<.05]$. Taking each observer separately, the difference was significant at the 5\% level for each of the four observers by the $Z$ test of Marascuilo $(1970$, p. 240). Conversely, sensitivity declined for three of the observers in the $(\mathrm{H}, \mathrm{V})$ and $(\mathrm{V}, \mathrm{H})$ conditions, in which mean $\mathrm{d}^{\prime}$ was .76 , although this effect is much smaller and not significant.

\section{Discussion}

The new finding in Experiment 3, not to the author's knowledge previously reported, was that sensitivity could be increased by a congruent image, that is, in the $(\mathrm{H}, \mathrm{H})$ and $(\mathrm{V}, \mathrm{V})$ conditions. To ensure that this increase was reliable, an abbreviated version of Experiment 3 was performed on four more observers. They ran through the same procedure, but were given only the $(\mathrm{H}, \mathrm{H}),(\mathrm{V}, \mathrm{V})$, (none, $\mathrm{H}$ ), and (none, $\mathrm{V}$ ) conditions. Mean $\mathrm{d}$ 's were 2.03 in the congruent conditions, $(\mathrm{H}, \mathrm{H})$ and $(\mathrm{V}, \mathrm{V})$, and .94 in the no-image conditions, replicating the earlier results.

Why should sensitivity increase with a congruent image? This result seems to oppose the usual finding that imagery reduces visual sensitivity (Segal, 1971). But this need not be so according to the limited-capacity notion. The limited-capacity channel is obliged to process target elements, even when these are irrelevant to the observer's decision (Experiment 2). However, the channel may process "stronger" target elements with higher priority than "weaker" ones. One of the possil' ways in which a target element might be "weakened" is by visual imagery. In the $(\mathrm{H}, \mathrm{H})$ condition, the image lies on top of the horizontal dot-pairs of the target and thus weakens them, leaving the critical dot-pair relatively unscathed to claim higher priority processing. Only in $(\mathrm{H}, \mathrm{H})$ and $(\mathrm{V}, \mathrm{V})$ can the image have this effect.

If images can take up processing capacity, then the incongruous image in the $(\mathrm{H}, \mathrm{V})$ and $(\mathrm{V}, \mathrm{H})$ conditions should lower sensitivity below the baseline conditions. For example, the $\mathrm{H}$ image in $(\mathrm{H}, \mathrm{V})$ should take capacity from the horizontally oriented critical dot-pair (in the $50 \%$ of $\mathrm{V}$ targets with a horizontally oriented critical 
dot-pair). Mean d's were .76 in $(\mathrm{H}, \mathrm{V})$ and $(\mathrm{V}, \mathrm{H})$ and .88 in the baseline condition, which is in the correct direction but is not a large enough effect to be perhaps wholly satisfactory.

This interpretation of imagery depends on the view that the patterned mask in the BM limits the duration of processing of the target in the second stage, thus allowing the observers to process only up to a fixed number of target dots, as shown by Experiment 1 . Observers in all three experiments reported that the targets appeared bright but very brief in BM, relative to their degraded but long-lasting appearance in NM, indirectly supporting this view (Liss, 1968). Very different results, showing parallel independent processing, may be obtained if dot masks are used which either produce a finely textured noise field or are presented as briefly as the target. In these cases, the mask appears to summate with the target to yield a composite percept in which the target is still visible for approximately its normal duration, but in a degraded form. Schultz and Eriksen (1977) provide excellent evidence for this sort of integration between target and mask, when both are around $8 \mathrm{msec}$ in duration. In the present research, mask duration was chosen to be long $(200 \mathrm{msec})$ relative to target duration $(20 \mathrm{msec})$, and so targets were not sufficiently energetic to alter the appearance of the mask through summation. At a zero SOA, the target could not be discriminated from a blank card in BM at better than chance accuracy by any of the observers tested in any of the three experiments. Hence, whatever summation occurred was insufficient to provide the observer with any information concerning target identity.

Both Experiment 2 and Experiment 3 demonstrate selective effects of visual imagery which depend on the relationship between the image and the target. These results provide evidence (rather circuitously) that observers are capable of maintaining images whose properties may be varied qualitatively in a systematic manner, although such abilities are unlikely to be universal (Richardson, 1969).

\section{REFERENCES}

GaRDNER, G. T. Evidence for independent parallel channels in tachistoscopic perception. Cognitive Psychology, 1973, 4, 130-155.

HAYs, W. L. Statistics. New York: Holt, Rinehart and Winston, 1963.

KAHNEMAN, D. Method, findings, and theory in studies of visual masking. Psychological Bulletin, 1968, 70, 404-425.
Liss, P. Does backward masking by visual noise stop stimulus processing? Perception \& Psychophysics, 1968, 4, 328-330.

Marascuilo, L. A. Extensions of the significance test for oneparameter signal detection hypotheses. Psychometrika, 1970, 35, 237-243.

Perky, C. W. An experimental study of imagination. American Journal of Psychology, 1910, 21, 422-452.

Philips, W. A. On the distinction between sensory storage and short-term visual memory. Perception \& Psychophysics, 1974, 16, 283-290.

Reeves, A., \& Segal, S. J. Effects of visual imagery on visual sensitivity and pupil diameter. Perceptual and Motor Skills, 1973, 36, 1091-1098.

Richardson, A. Mental imagery. New York: Springer, 1969.

SChEERER, E. Integration, interruption and processing rate in visual backward masking. Psychologische Forschung, 1973, 36, 71-93.

Schultz, D. W., \& Eriksen, C. W. Do noise masks terminate target processing? Memory \& Cognition, 1977, 5, 90-96.

Segal, S. J. Imagery. New York: Academic Press, 1971.

SEgAl, S. J., \& Fusella, V. Influence of imaged pictures and sounds on detection of auditory and visual signals. Journal of Experimental Psychology, 1970, 83, 458-464.

SPe Rling, G. A model for visual memory tasks. Human Factors, 1963, 5, 19-31.

Treisman, M. On the stability of $d_{s}$. Psychological Bulletin, 1977, 84, 235-243.

TuRvey, M. T. On peripheral and central processes in vision: Inferences from an information-processing analysis of masking with patterned stimuli. Psychological Review, 1973, 80, 1-52.

UTTAL, W. R. An autocorrelation theory of form detection. New York: Wiley, 1975.

\section{NOTES}

1. A two-way fixed-effects ANOVA (Hays, 1963) showed significant effects of masking condition, target numerosity (numerosity 4 was excluded, as it had been equated for observers and conditions by the design), and their interaction $[F(1,24)=270, F(3,24)$ $=713$ and $F(3,24)=42.6$, respectively]. They accounted for $10 \%$, $83 \%$, and $5 \%$ of the variance, respectively, using the index $\omega^{2}$ (Hays, 1963, p. 407).

2. In the two-way ANOVA, the four imagery levels were none, image mask, image same, and image different. The latter two levels were constructed artificially, because the same vs. different distinction has meaning only in the separate target set conditions. The levels were defined so that "same" referred to $(\mathrm{H}, \mathrm{H})$ and $(\mathrm{V}, \mathrm{V})$ in the separate target set and to $(\mathrm{H}, \mathrm{H}+\mathrm{V})$ in the mixed; and "different" referred to $(\mathrm{H}, \mathrm{V})$ and $(\mathrm{V}, \mathrm{H})$ in the separate target set and to $(\mathrm{V}, \mathrm{H}+\mathrm{V})$ in the mixed. This method ensured that the design was mathematically factorial and that each $\mathrm{d}^{\prime}$ was based on the same number of trials. Given that imagery produced a significant main effect, it was then permissible to use planned comparisons to contrast any of the four levels in the separate target set, where they have a natural meaning.

(Received for publication November 9, 1979; revision accepted March 25, 1980.) 